embryo (H. Meinhardt, Max Planck Institut fur Entwicklungsbiologie, Tübingen). There are three zygotic gap genes, which differ with respect to the region of the embryo affected by mutations of them: hunchback mutations delete thoracic segments; knirps mutations delete abdominal segments; and Kruppel mutations delete thoracic and anterior abdominal segments. It seems that in the absence of the appropriate gap gene, large regions of the embryo simply fail to develop, the remaining segments developing normally. Further insight into their workings should be forthcoming, now that the Krüppel gene has been cloned (H. Jäckle, Max Planck Institut fur Entwicklungsbiologie, Tübingen). The partial rescue of mutant embryos by injecting the cloned DNA into the prospective thoracic and anterior abdominal region suggests that the product of the Krüppel gene is active in the region of the embryo affected by Krüppel mutations. The major RNA transcript detected by hybridization with the cloned Krüppel DNA is expressed specifically at the blastoderm stage of development, which is the time when the Krüppel gene product seems to be required.

By the time that signs of segmentation first become apparent in the life of a fruitfly, the embryo's nuclei have become transcriptionally active and its development is no longer dominated by its maternal inheritance. However, although most of the segmentation genes are strictly zygotic, several of the knirps group of gap genes, including oskar, are actually maternaleffect genes. Nüsslein-Volhard (FriedrichMiescher Laboratorium, Tübingen) reported that the phenotype of oskar mutant embryos (which would normally fail to develop an abdomen) can be completely rescued by the injection of wildtype cytoplasm as long as the cytoplasm is taken from the posterior pole of a wild-type embryo and injected into the prospective abdominal region of the mutant embryo. The implication is that the rescuing activity is actually stored at the posterior pole and transported anteriorly, in some way influencing the development of cells in the abdominal region of the embryo.

Of the genes that have a periodic effect on the segmentation pattern when mutated, most is known of the pair-rule gene fushi tarazu ( $f t z)$. In homozygous $f t z$ mutant embryos, roughly the posterior half of every even-numbered segment and the anterior half of every odd-numbered segment is deleted; the remaining halfsegments are fused, so that the mutant embryos have half the usual number of segments. Interestingly, in normal embryos, ftz RNA is distributed periodically, with a periodicity roughly corresponding to that of the deleted parts of $\mathrm{ftz}$ mutant embryos, before the first morphological signs of segmentation (A. Kuraiwa, Biozentrium, Basel; refs 2, 11, 12).

Another pair-rule gene, Hairy, seems to be the complement of $f t z$ in that Hairy

\section{Homoeo box latest}

WALTER Gehring revealed at the European Developmental Biology Congress in Southampton two weeks ago that his group in Basle, in collaboration with Tom Kornberg in San Francisco, has now identified the first gene in Drosophila outside of the bithorax and Antennapedia complexes to contain a homoeo box sequence. The gene is engrailed, which keeps up the association of the homoeo box with genes that specify the development of different parts of the fruitfly as engrailed is also a homoeotic gene, with the particular role of distinguishing the posterior from the anterior compartments of each segment. Gehring also reported that it is now clear that at least nine homoeo boxes fall within the bithorax and Antennapedia complexes: three have been mapped within the bithorax complex (two by Welcome Bender's group at Harvard), and there are at least six in the Antennapedia complex, of which three have been assigned to known genes (see the figure; note that further genes are believed to exist within Antennapedia, left of $p b$ ).

The disposition of the three homoeo boxes within the bithorax complex may be especially significant in the light of some recent results of Gines Morata (University of Madrid). Morata reported at the Crete meeting that on the basis of complementation analyses the bithorax complex seems to consist of three distinct genes: Ultrabithorax $(U b x)$, abdominal- $A$

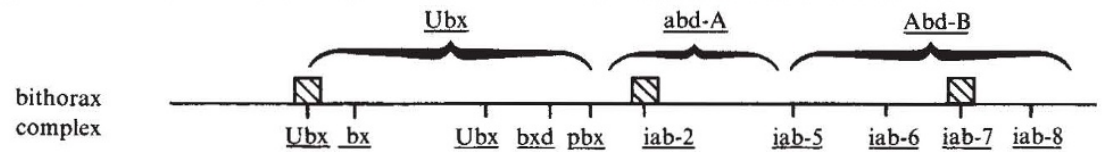

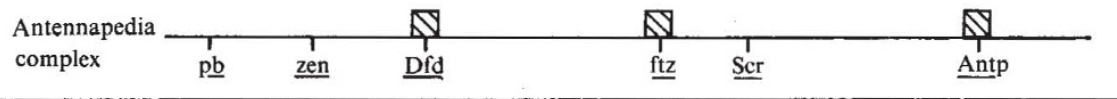

mutations lead to the deletion of the parts not deleted in $\mathrm{ftz}$ mutants. An important point made by D. Ish-Horowicz (Imperial Cancer Research Fund, London) is that the parts of the embryo not deleted in Hairy mutants retain their wild-type segment identity. By combining Hairy and homoeotic mutations it has also been shown that the requirement for a wild-type Hairy gene depends upon the position of a group of cells in the embryo, and not upon the character of the segment of which the cells will form a part. So it would seem that segment specification - the domain of the homoeotic genes - and whatever genes such as $f t z$ and Hairy are doing, are to some extent independent processes. IshHorowicz revealed that the Hairy gene has been cloned and shown to be expressed early in development, so it will be interesting to see how the spatial distribution of Hairy transcripts compares with that of $f t z$ transcripts.

\section{Specifying the parts}

The importance as units of development of the parts into which segmentation divides the body of a fruitfly is clearly shown by the effects of homoeotic mutations, which cause specific parts of the body to develop $(a b d-A)$ and $A b d o m i n a l-B(A b d-B)$. Each of these genes is concerned with specifying the development of a different region of the fruitfly: thus, the $U b x$ domain extends from the posterior compartment of the second thoracic segment to the anterior compartment of the first abdominal segment; the $a b d-A$ gene then takes over as far as the fourth abdominal segment; and the $A b d-B$ gene specifies the four most posterior segments. As can be seen in the figure, the three homoeo boxes have fallen neatly one into each genetically defined unit.

Although evidence is still lacking that the highly-conserved homoeo box sequences that have been found in the genomes of vertebrates really are markers of genes involved in regulating development, the assumption has now received further support from the first studies of the expression of such a gene. The crucial finding reported by Eddy de Robertis (Basle) in Southampton is that the second homoeo box-containing gene his group has identified in the genome of the frog Xenopus laevis is expressed at high levels in oocytes, and that transcripts of the gene disappear in early (blastula-phase) embryos. Transcripts of the gene can again be detected at gastrulation, but at a much lower level than in oocytes. The exciting possibility suggested by these observations is that they have identified a gene that encodes a stored maternal mRNA that may be involved in specifying the early development of the frog embryo. affected parts are usually delineated by either two segment boundaries, or a segment boundary and a line that divides a segment into anterior and posterior halves. Homoeotic mutations are thought to identify genes that specify the development of different parts of a fruitfly's body, and their domains of action are known as developmental compartments.

The archetypal homoeotic genes cluster in a region of the Drosophila genome known as the bithorax complex, which controls the development of all segments of the Drosophila body posterior to the second thoracic segments. Recessive bithorax complex mutations cause segments to develop like more anterior segments, and the genetic order of the mutants roughly corresponds to the spatial order of the affected segments along the anterior-posterior axis ${ }^{13}$.

These observations led E.B. Lewis of the California Institute of Technology to propose that, starting with the third thoracic segment, progressively more posterior segments express successively more of the genetic components of the bithorax complex, and that the particular combination of genes expressed by groups 